\title{
Posterior fossa abnormalities in high-risk term infants: comparison of ultrasound and MRI
}

\author{
S. J. Steggerda • F. T. de Bruïne • V. E. H. J. Smits-Wintjens • \\ P. Verbon • F. J. Walther • G. van Wezel-Meijler
}

Received: 5 September 2014 / Revised: 29 January 2015 / Accepted: 12 February 2015 / Published online: 22 April 2015

(C) The Author(s) 2015. This article is published with open access at Springerlink.com

\begin{abstract}
Objectives We aimed to assess the characteristics of posterior fossa (PF) abnormalities in a cohort of high-risk term neonates, as well as the diagnostic performance of cranial ultrasound (CUS) with additional mastoid fontanelle (MF) views for the detection of these abnormalities, with magnetic resonance imaging (MRI) being the reference standard.

Methods In this retrospective study, 113 term neonates with CUS and subsequent MRI were included. Sensitivity, specificity, and predictive values of routine CUS and CUS with MF views were calculated.

Results Posterior fossa abnormalities were diagnosed on CUS in 46 of 113 infants. MRI confirmed these findings in 43 and showed additional abnormalities in 32 infants. The sensitivity and specificity of anterior fontanelle views for major PF abnormalities as seen on MRI were $16 \%$ and $99 \%$. Adding MF views increased the sensitivity of US to $82 \%$. The sensitivity and specificity of MF views for the detection of any (major or minor) PF abnormality were $57 \%$ and $95 \%$. Especially acute hypoxic-ischemic injury and small subdural and punctate cerebellar haemorrhage remained undetected by CUS.

Conclusions PF abnormalities are frequent in high-risk term infants. MF-CUS enables early diagnosis of major PF
\end{abstract}

S. J. Steggerda $(\varangle) \cdot$ V. E. H. J. Smits-Wintjens $\cdot$ P. Verbon •

F. J. Walther

Department of Neonatology, Leiden University Medical Centre,

Leiden, The Netherlands

e-mail: s.j.steggerda@lumc.nl

F. T. de Bruïne • G. van Wezel-Meijler

Department of Radiology, Leiden University Medical Centre,

Leiden, The Netherlands

G. van Wezel-Meijler

Department of Neonatology, Isala Hospital, Zwolle, The Netherlands abnormalities. We therefore advocate to perform MF-CUS in high-risk term neonates.

Key Points

- Posterior fossa abnormalities are a frequent finding in highrisk term infants.

- Adding mastoid fontanelle views improves ultrasound detection of clinically relevant abnormalities.

- Hypoxic-ischemic injury and small posterior fossa haemorrhages are better detected with MRI.

- Cranial ultrasound examination should include mastoid fontanelle views in high-risk term neonates.

Keywords Infant, newborn · Ultrasound $\cdot$ MRI $\cdot$ Posterior fossa $\cdot$ Cerebellum

\section{Abbreviations}

AF Anterior fontanelle

CUS Cranial ultrasound

CNS Central nervous system

TE Echo time

FOV Field of view

HIE Hypoxic-ischemic encephalopathy

MRI Magnetic resonance imaging

MF Mastoid fontanelle

$\mathrm{PF} \quad$ Posterior fossa

TR Repetition time

SI Signal intensity

\section{Introduction}

The current widespread availability of advanced neuroimaging techniques has led to the increased detection of cerebellar injury and other posterior fossa (PF) abnormalities in newborn 
infants requiring intensive care. Cerebellar injury is an important complication of preterm birth and may also occur in fullterm infants $[1,2]$.

In full-term infants, cerebellar and extra axial PF haemorrhage is associated with traumatic delivery, perinatal infection, supratentorial haemorrhage and perinatal asphyxia [2-4]. The cerebellum can be involved in hypoxic-ischemic injury [5]. Cerebellar abnormalities are reported in neonates with metabolic disorders [6, 7], and congenital PF malformations are common in neonates with other central nervous system (CNS) abnormalities.

Cerebellar abnormalities are associated with a broad spectrum of neurodevelopmental disabilities [2, 8]. Early detection is therefore important. Cranial ultrasound (CUS) is the primary neonatal neuroimaging technique. It can be performed early and repeatedly at the bedside, and provides instant diagnostic information. CUS is traditionally performed through the anterior fontanelle (AF). This enables reliable views of supratentorial structures, but visualization of the PF is less optimal due to its distance away from the transducer [9]. In addition, the echogenic tentorium and vermis may impede visualization. The mastoid fontanelle (MF) enables a better view of the PF [10-12]. A previous study [13] demonstrated that addition of MF views in preterm infants improved detection of cerebellar injury as compared to CUS with only AF views. Despite the clinical importance of cerebellar injury and other PF abnormalities, MF views are often not included in the CUS examination of the brains of high-risk term infants.

The posterior fossa is well depicted on magnetic resonance imaging (MRI). However, compared with CUS, MRI is a burdensome procedure in critically ill neonates and is less suitable for early or serial imaging.

The aims of this study were to assess: (1) the frequency and characteristics of PF abnormalities in a cohort of high-risk term neonates, and (2) the diagnostic performance of CUS with additional MF views (MF-CUS) for the detection of these abnormalities, with MRI being the diagnostic reference standard.

\section{Materials and methods}

Patients

The ethics committee of the Leiden University Medical Centre waived the need for informed consent for this retrospective study. A systematic database search identified all term infants (gestational age $>36$ weeks) admitted to the neonatal unit of the Leiden University Medical Centre (tertiary neonatal referral centre) between January 2008 and December 2012 with a diagnosis of hypoxic-ischemic encephalopathy (HIE), central nervous system (CNS) infection, supratentorial intracranial haemorrhage, ventricular dilatation, metabolic disorder, congenital CNS malformation and/or abnormal neurologic examination [3]. Infants were eligible for the study if they had undergone at least one good quality CUS that included both $\mathrm{AF}$ and MF views and at least one MRI examination during admission.

We included 113 infants with a total of 187 CUS examinations [mean number of CUS 1.7 per patient (range 1-7)] and 117 MR examinations. MF-CUS was performed at a mean age of 5.4 days $(\mathrm{SD} \pm 5.0)$, and MRI at a mean age of 8.0 days $(\mathrm{SD} \pm 8.8)$. The mean interval between MFCUS and MRI was 3.1 days ( $\mathrm{SD} \pm 7.9$ ). Patient characteristics are shown in Table 1.

\section{CUS}

CUS was performed by an experienced neonatologist or fellow-neonatologist, using an US system with multifrequency (5-10 MHz) transducers (Aloka Alpha 10; Biomedic Nederland BV, Almere, The Netherlands). Our standard CUS protocol includes imaging through the $\mathrm{AF}$ in at least six coronal

Table 1 Patient characteristics $(n=113)$

\begin{tabular}{lc}
\hline Gestational age (weeks)* & $38.8 \pm 1.7$ \\
Birth weight (grams)* & $3,280 \pm 679$ \\
Male gender & $61(54)$ \\
Mode of delivery & \\
$\quad$ Spontaneous birth & $46(41)$ \\
Vacuum/forceps assisted & $22(20)$ \\
Elective caesarean section & $18(16)$ \\
Emergency caesarean section & $27(24)$ \\
Apgar score 5 min\# & $5.8(0-10)$ \\
Died & $22(20)$ \\
High risk category: & \\
HIE & $51(45)$ \\
Haemorrhage & $10(9)$ \\
Traumatic delivery & $4(4)$ \\
CNS infection & $5(4)$ \\
Metabolic disorder & $8(7)$ \\
Congenital malformation & $18(16)$ \\
Other & $17(15)$ \\
Age at MF-CUS (days)* & $5.4 \pm 5.0$ \\
Age at MRI (days)* & $8.0 \pm 8.8$ \\
- Cases with HIE (days)* & $6.3 \pm 1.4$ \\
Number of MF-CUS\# & $1.7(1-7)$ \\
\hline HIE = hypoxic-ischemic encephalopathy, CNS = central nervous system, \\
MF-CUS = mastoid fontanel cranial ultrasound \\
Except when indicated, data are number of infants with percentages in \\
parentheses \\
* mean \pm standard deviation \\
\# mean, with range in parentheses \\
$\quad$ \\
\hline
\end{tabular}


and five sagittal planes [14]. The standard transducer frequency is set at $7.5 \mathrm{MHz}$. To assess deeper structures, including the posterior fossa, a frequency of $5 \mathrm{MHz}$ can be applied. Depending on the clinical history of the infant and the imaging findings on the AF views, the sonographer can decide to perform additional MF views in term neonates who are considered at risk for PF abnormalities (neonates with HIE, CNS infection, intracranial haemorrhage, ventricular dilatation, metabolic disorder, congenital CNS malformation and/or abnormal neurologic examination) [3]. Scanning through the MF is performed in axial and coronal planes (Fig. 1), with views of the ventricular system, brain stem, cerebellar peduncles, vermis, and hemispheres $[9,11,14]$. Generally, one of the MF is used, depending on the position of the infant. When abnormalities are suspected, additional images can be obtained using the opposite MF.

\section{MRI}

In our hospital, MRI examination of the brain (Achieva $3 \mathrm{~T}$; Philips Medical Systems, Best, The Netherlands) is performed in all neonates with clinical or CUS suspicion of parenchymal brain abnormalities. Details of the MRI procedure and imaging protocols have been reported previously [15]. A standard MRI examination includes a 3D T1-weighted turbo field-echo sequence, a T2-weighted turbo spin-echo sequence, a T2* fast field-echo sequence, and diffusion-weighted spin-echo sequences in three directions.

All CUS images that included both $\mathrm{AF}$ and MF views were reviewed by at least two investigators (GM has 25 years of experience, SS and VSW have 10 years of experience in CUS). To avoid recall bias, the scans were reviewed at least 4 months after they were made. The MRI images were also reviewed by two experienced investigators $(\mathrm{FdB}$, paediatric neuroradiologist with 20 years of experience and SS, with 10 years of experience in neonatal MRI). This was done at least 6 weeks after reviewing the CUS images. The investigators were blinded to the patients' names and clinical history and reviewed the images together. Any discrepancies in interpretation were resolved by consensus.

CUS and MRI images were reviewed for the presence of PF malformations, loss of normal anatomic features, local or diffuse echogenicity changes or signal intensity (SI) changes in the cerebellar parenchyma (hemispheres and/or vermis), abnormalities in size and/or shape of the fourth ventricle, haemorrhage within the fourth ventricle, extra axial posterior fossa haemorrhage, and other abnormalities in PF structures (cisterna magna, subarachnoid and subdural spaces). When abnormalities were present, location and extent were noted, and if abnormalities were seen on MRI, it was noted whether these were visible on T1-weighted and/or T2-weighted, or only on $\mathrm{T}_{2}{ }^{*}$ or diffusion-weighted images.

The following US and MRI abnormalities were considered major and possibly clinically relevant: PF malformation, large cerebellar haemorrhage ( $>4 \mathrm{~mm}$ ), diffuse echogenicity or SI changes in the cerebellar parenchyma, large extra axial haemorrhage with mass effect, and fourth ventricular dilatation or compression. Other abnormalities, such as mild enlargement of the cisterna magna with an intact vermis and without other structural abnormalities or mass effect, punctate cerebellar haemorrhage $(<4 \mathrm{~mm})$, and small subdural or subarachnoid haemorrhage without mass effect, were considered minor and less relevant, based on available literature [16-18].

\section{Data analysis}

Statistical analysis was performed using SPSS software (SPSS 17; Chicago, Illinois, USA). The frequency of PF abnormalities on CUS and MRI was calculated. We compared the diagnostic performance of CUS for the detection of PF abnormalities with the results of MRI, which was considered to be the reference standard. We calculated sensitivity, specificity, positive predictive value and negative predictive value of CUS, with and without MF views.

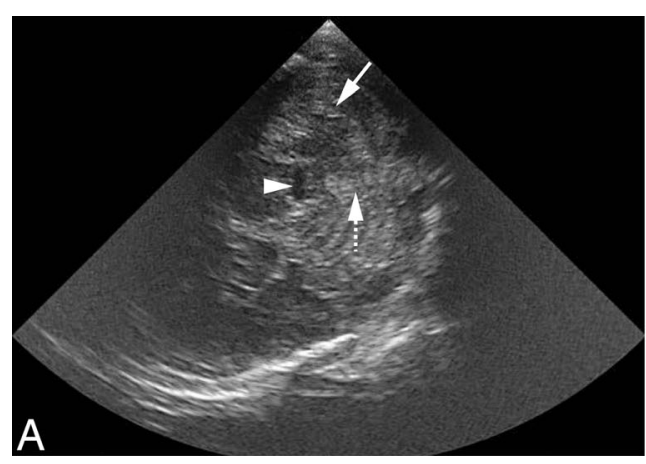

Fig. 1 Normal ultrasound in healthy term neonate using the MF as acoustic window. $(A)$ Axial and $(B)$ coronal views (third postnatal day, right $\mathrm{MF}$ ) showing normal cerebellar hemispheres [the hemisphere

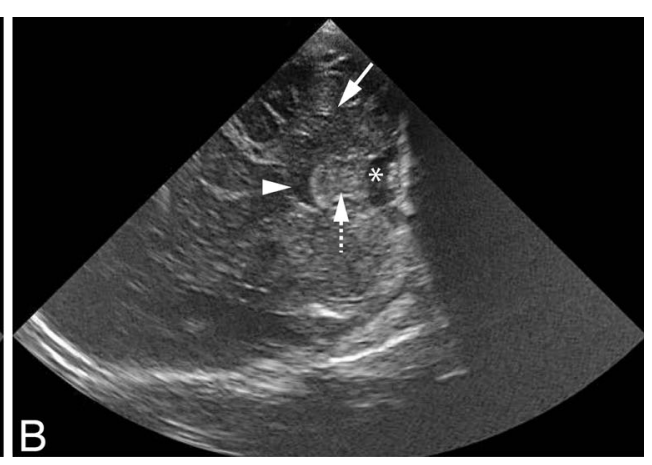

located closest to the transducer is visualized in the upper part of the image (arrow)], vermis (dotted arrow), fourth ventricle (arrowhead) and cisterna magna (asterix) 


\section{Results}

In $46(41 \%)$ of the 113 infants, CUS detected abnormalities in the PF. In 39 (85\%) of 46 infants, these abnormalities were seen only on MF views. In six infants (13\%), abnormalities were seen on both AF and MF views, and in one infant $(2 \%)$, abnormalities were seen only on AF views.

In $75(66 \%)$ of the 113 infants, MRI depicted abnormalities in the PF.

\section{Comparison CUS and MRI}

\section{MRI findings in infants with abnormal CUS}

In the only infant in whom a PF abnormality was suspected on the AF, but not on the MF views, no PF abnormality was seen on MRI. In 43 (96\%) of 45 infants, the PF abnormalities on MF-CUS were confirmed by MRI. In 31 infants, the abnormalities were classified as major (Table 2), including two infants with a large extra axial haemorrhage, two with a large cerebellar haemorrhage and three with a haemorrhagic clot in the fourth ventricle and post-haemorrhagic ventricular dilatation (Fig. 2). In nine infants with HIE, in whom MF-CUS showed echogenicity changes of the cerebellar vermis and/or hemispheres, MRI showed SI changes on T1_/T2-weighted and/or diffusion-weighted images (Fig. 3). In two of these cases, MRI showed additional small haemorrhagic lesions in the cerebellar parenchyma. In three infants with CNS infection, PF abnormalities were seen on both MF-CUS and MRI (Fig. 4). There were six cases with cerebellar hypoplasia (Fig. 5); in five of them, a metabolic disorder was diagnosed. Six infants had other major PF malformations diagnosed on both MF-CUS and MRI (Table 2).
In 12 infants, minor abnormalities were diagnosed on both MF CUS and MRI: punctate cerebellar haemorrhage (one infant) (Fig. 6), small extra axial haemorrhage (nine infants), and mildly enlarged cisterna magna (two infants).

In two infants, the abnormalities suspected on MF-CUS were not confirmed by MRI. One infant with meningitis showed increased echogenicity and loss of foliation of both cerebellar hemispheres on MF-CUS, possibly representing oedema (classified as major, Table 3 ). The MRI performed 8 days later showed no abnormalities. In the other infant, a minor extra axial haemorrhage was suspected on MF-CUS, but in retrospect, this was a misinterpretation of an imaging artefact and might have been avoided when both MF had been used.

\section{MRI findings in infants with normal CUS findings}

MRI detected abnormalities in 32 infants with normal CUS findings. In seven infants, these abnormalities were classified as major (Table 3): in four infants with HIE, MRI showed SI changes of the cerebellar vermis, which were only seen on the diffusion-weighted images (Fig. 7); in one infant with alloimmune thrombocytopenia, T2* MRI sequences showed a haemorrhagic lesion in the cerebellar vermis, probably of foetal origin; one infant had cerebellar dysplasia with abnormal foliation, which was, in retrospect, also visible on MF-CUS; and one infant had a small arachnoid cyst in the cerebellopontine angle, which could not be visualized on the MF views. The other 25 infants had minor PF abnormalities, including punctate cerebellar haemorrhage (three infants), small extra axial haemorrhage (18 infants), and combined punctate cerebellar haemorrhage and small extra axial haemorrhage (four infants).

Table 2 Imaging findings in 31 infants with major abnormalities on MF-CUS, confirmed with MRI

\begin{tabular}{|c|c|c|c|}
\hline Category & MF-CUS findings & MRI findings & No infants \\
\hline HIE & $\begin{array}{l}\text { Increased echogenicity cerebellar vermis } \\
\text { and hemispheres }\end{array}$ & $\begin{array}{l}\text { Abnormal SI cerebellar vermis and } \\
\text { hemispheres (also punctate CBH) }\end{array}$ & $9(2)$ \\
\hline \multirow[t]{2}{*}{ CNS infection } & $\begin{array}{l}\text { Increased echogenicity cerebellar vermis } \\
\text { and hemispheres }\end{array}$ & $\begin{array}{l}\text { Abnormal SI cerebellar vermis and } \\
\text { hemispheres }\end{array}$ & 2 \\
\hline & $\begin{array}{l}\text { Subdural debris, dilated fourth ventricle } \\
\text { with debris }\end{array}$ & $\begin{array}{l}\text { Subdural debris, dilated fourth ventricle } \\
\text { with debris }\end{array}$ & 1 \\
\hline Metabolic disorder & Cerebellar hypoplasia & Cerebellar hypoplasia & 5 \\
\hline \multirow[t]{3}{*}{ CNS malformation } & Cerebellar hypoplasia & Cerebellar hypoplasia & 1 \\
\hline & $\begin{array}{l}\text { Arachnoid cyst with mass effect on } \\
\text { cerebellum }\end{array}$ & $\begin{array}{l}\text { Arachnoid cyst with mass effect on } \\
\text { cerebellum }\end{array}$ & 3 \\
\hline & $\begin{array}{l}\text { Displacement of PF structures, abnormal } \\
\text { fourth ventricle }\end{array}$ & $\begin{array}{l}\text { Displacement of PF structures, abnormal } \\
\text { fourth ventricle }\end{array}$ & 3 \\
\hline \multirow[t]{3}{*}{ PF haemorrhage } & $\begin{array}{l}\text { Echogenic clot in dilated fourth ventricle, } \\
\text { PHVD }\end{array}$ & $\begin{array}{l}\text { Echogenic clot in dilated fourth ventricle, } \\
\text { PHVD }\end{array}$ & 3 \\
\hline & $\begin{array}{l}\text { Large extra axial PF haemorrhage with } \\
\text { mass effect on cerebellum and brain stem }\end{array}$ & $\begin{array}{l}\text { Large extra axial PF haemorrhage with } \\
\text { mass effect on cerebellum and brain stem }\end{array}$ & 2 \\
\hline & Major cerebellar haemorrhage & Major cerebellar haemorrhage & 2 \\
\hline
\end{tabular}



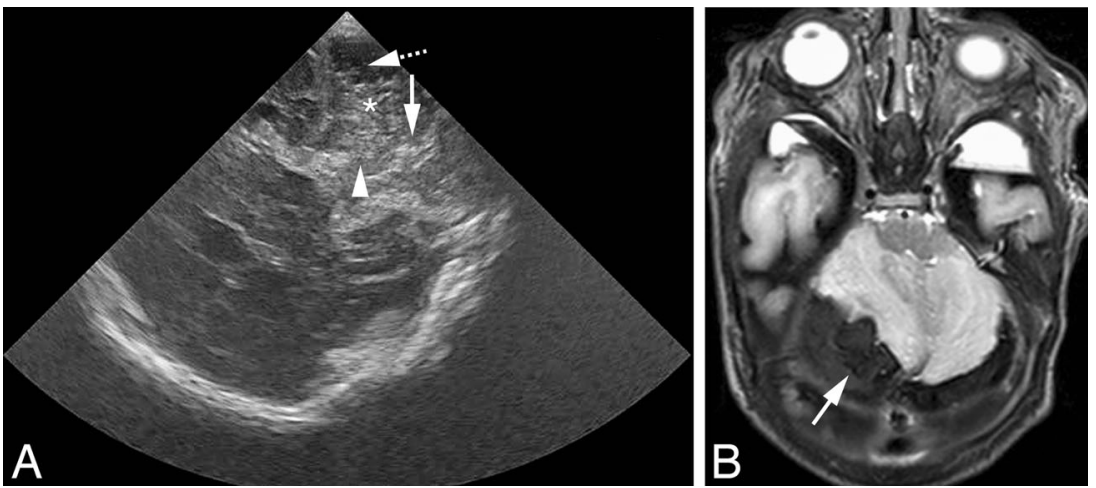

Fig. 2 Male infant born at 37 weeks gestational age after precipitous labour. Abnormal neurologic examination and respiratory failure developing in the first hours after birth. $(A)$ Coronal CUS (second postnatal day, right MF) shows a large extra axial haemorrhage (dotted arrow) with mass effect on the right cerebellar hemisphere (asterix), shift

\section{Predictive values of CUS}

The sensitivity and specificity of routine AF-CUS views for major PF abnormalities, as seen on MRI, were $16 \%$ and $99 \%$, and the positive and negative predictive values were $86 \%$ and $70 \%$, respectively. Adding the MF views did not change the specificity of CUS for major PF abnormalities, but increased the sensitivity to $82 \%$ and the positive and negative predictive values to $97 \%$ and $91 \%$, respectively.

The sensitivity and specificity of CUS with additional MF views for the detection of any (major or minor) PF abnormality, as seen on MRI, were $57 \%$ and $95 \%$. The positive predictive and negative predictive values were $96 \%$ and $53 \%$, respectively.

\section{Discussion}

This is the first study assessing the prevalence of PF abnormalities in high-risk term infants and comparing the of the vermis (arrow) and compression of fourth ventricle (arrowhead). (B) T2-weighted MRI on the same day confirms the presence of a large extra axial posterior fossa haemorrhage (arrow) with mass effect on the cerebellum and brain stem. Emergent neurosurgical decompression was performed and the boy fully recovered

diagnostic accuracy of CUS, including both AF and MF views, with MRI.

MRI detected PF abnormalities among 75 (66\%) of the 113 infants in our cohort of high-risk term infants; in 38 (34\%) of the infants, these abnormalities were classified as major. The majority ( $82 \%$ ) of these abnormalities were also found on MF views, but remained undetected when only AF views were used. In seven infants, MRI demonstrated major $\mathrm{PF}$ abnormalities that remained undetected on MF views.

Our results indicate that the addition of MF views to standard AF-CUS can be used as an early diagnostic tool in highrisk term infants. Most infants in our study had a clinical picture and/or supratentorial CUS abnormalities that would eventually require MRI examination. However, due to practical shortcomings such as transportation of the neonate, MRI can be undesirable or unfeasible during the first days after birth, especially in critically ill neonates. The strength of MF-CUS is that it takes only a few minutes of extra scanning time, and in experienced hands, detects the majority of major PF abnormalities much earlier than possible with MRI.
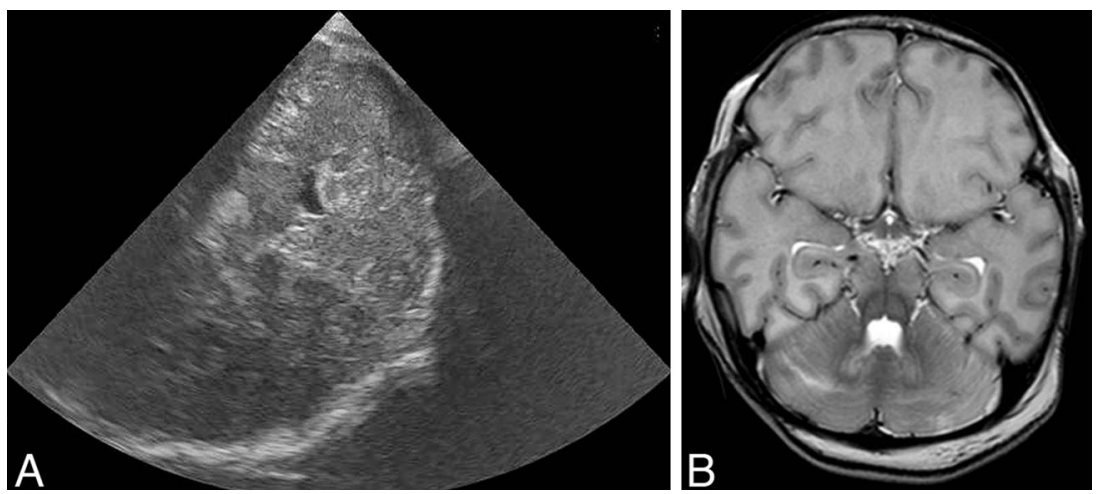

Fig. 3 Female infant born at 41 weeks gestational age, with severe perinatal asphyxia. (A) Axial CUS scan (fifth postnatal day, left MF) shows diffuse increased echogenicity of the cerebellum with loss of normal appearance of cerebellar foliae. (B) T2-weighted MR image on

same day shows abnormal signal intensity in both cerebellar hemispheres with loss of normal foliation, and also shows extensive supratentorial signal intensity changes in the cerebral hemispheres 

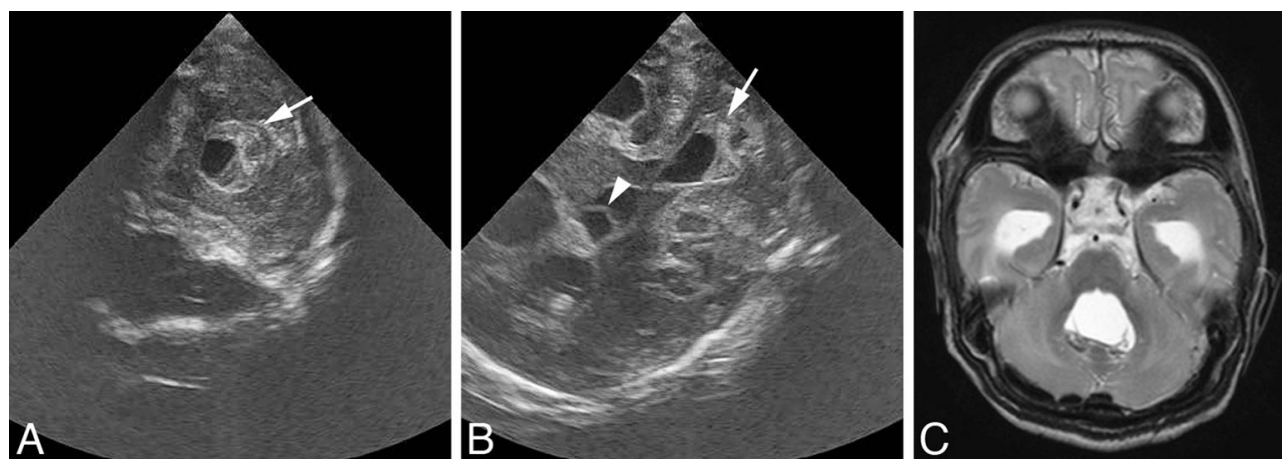

Fig. 4 Male infant born at 41 weeks gestational age, admitted at eight postnatal day with E. Coli meningitis and symptoms of increased intracranial pressure. CUS scan though $\mathrm{AF}$ showed severe ventricular dilatation (not shown). (A) Axial and (B) coronal ultrasound (eight

The high percentage of infants with PF abnormalities in this study $(66 \%)$ is probably secondary to the inclusion of both major and minor abnormalities in a high-risk population. Furthermore, we used diffusion-weighted images to detect acute hypoxic-ischemic injury and $\mathrm{T} 2 *$ sequences that are especially sensitive for the detection of small hemosiderin deposits [19]. In addition, T1-weighted and T2-weighted images had a slice thickness of 1 and $2 \mathrm{~mm}$ respectively, thereby assuring the detection of even the smallest lesions.

The most frequent major abnormalities that were detected were SI changes in the cerebellar parenchyma in cases with severe HIE. In about two-thirds of these cases, abnormalities (increased echogenicity or loss of normal architecture) were also diagnosed on MF-CUS views. However, especially acute hypoxic ischemic injury of the cerebellar vermis as seen on diffusion-weighted MR images remained undetected on CUS.

The high incidence of cerebellar abnormalities in infants with HIE is not consistent with some previous studies reporting sparing of the cerebellum in term infants with HIE [20-22]. Other studies, however, have shown involvement of postnatal day, left MF) showing debris in dilated fourth ventricle (arrow) and strands in third ventricle (arrowhead). (C) T2-weighted MRI on the same day confirms CUS findings

the cerebellum in neonates with hypoxic-ischemic brain injury [23-28]. Previous neuroimaging studies that used late or follow-up MR scans, or autopsy and combined autopsy/MR studies described involvement of the cerebellum (in particular the vermis) in HIE [24-28]. Cerebellar abnormalities were also reported in $13 \%$ of full term infants with HIE and basal ganglia-thalamic (BGT) injury [23]. The higher incidence of hypoxic-ischemic cerebellar injury in our study may be explained by the frequency of this finding on the diffusionweighted MR images, which were not included in the former study. The infants with HIE and cerebellar injury in our study frequently had severe BGT and/or brain stem injury, and had a high mortality $(62 \%)$. The former study also reported high mortality and poor neurologic outcome of infants with cerebellar involvement. We therefore assume that cerebellar injury mainly occurs in neonates with severe HIE brain injury. This assumption needs to be confirmed by larger studies. However, since cerebellar injury in HIE mainly seems to occur in the most severe cases, it is unclear whether this finding will have an independent effect on outcome [23]. Future studies
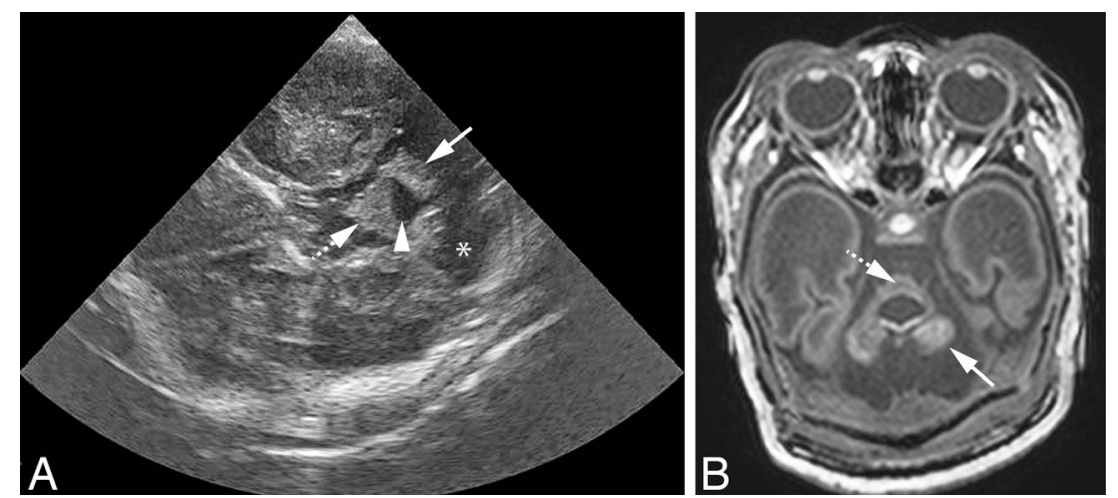

Fig. 5 Male infant born at 38 weeks gestation who died in the neonatal period. Foetal polyhydramnios, neonatal hypotonia, contractures and respiratory failure. Suspected type IV 3-Methylglutaconic aciduria. AFCUS showed a dilated ventricular system with abnormal echogenicity of the basal ganglia and abnormal gyral pattern. $(A)$ Axial CUS scan (third postnatal day, right MF) shows severe hypoplasia of cerebellum (arrow) and pons (dotted arrow), with enlarged fourth ventricle (arrowhead) and pericerebellar spaces (asterix). (B) T1-weighted MRI confirms severely hypoplastic cerebellum (arrow) and pons (dotted arrow). Gyral abnormalities of the temporal lobes can be seen on this image 

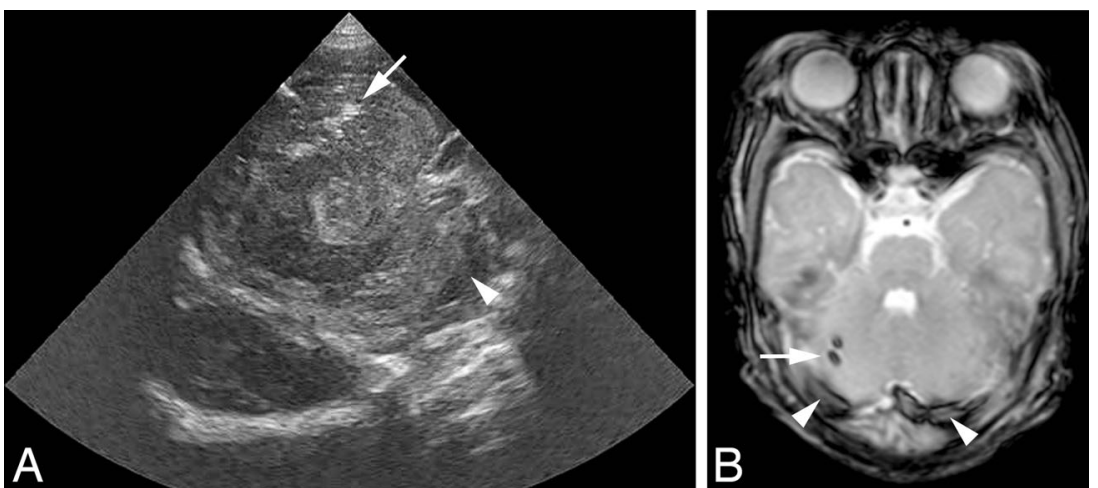

Fig. 6 Male infant born asphyxiated at 39 weeks gestational age after non-assisted vaginal delivery. $(A)$ Axial CUS (fifth postnatal day, right MF) shows small focal echogenic lesions in right cerebellar hemisphere (arrow), suspect for haemorrhages and small extra axial haemorrhage around left cerebellar hemisphere (arrowhead). (B) T2*-weighted MRI (seventh postnatal day) confirms small cerebellar haemorrhages in right hemisphere (arrow) and small extra axial posterior fossa haemorrhage (arrowheads) investigating cerebellar injury in HIE and its consequences on cerebellar development and neurodevelopmental outcome in term infants are needed.

Another major abnormality was cerebellar hypoplasia, which was mostly detected in infants with inborn errors of metabolism. All cases of cerebellar hypoplasia could be diagnosed on MF-CUS views. Several inborn errors of metabolism can affect early cerebellar development $[6,7]$ and lead to cerebellar hypoplasia or atrophy. In combination with a clinical picture suggesting a metabolic disorder, this finding on CUS may help diagnose the underlying disease.

Apart from a haemorrhagic lesion in the cerebellar vermis that was probably of antenatal origin and was only detected on T2* MRI, all other major PF haemorrhages were detected on MF-CUS views. These included large cerebellar haemorrhage, large extra-axial haemorrhage with mass effect, and haemorrhage and dilatation of the fourth ventricle. The finding of large PF haemorrhage can be relevant, as these haemorrhages may cause cerebellar and brain stem compression, or progressive ventricular dilatation requiring neurosurgical intervention. In preterm infants, large cerebellar haemorrhage has important prognostic consequences. In term infants, these larger haemorrhages are less commonly reported, but they have also been associated with a broad spectrum of neurodevelopmental disabilities $[2,8]$.

The most common minor abnormalities seen were small extra axial haemorrhages in $34 \%$ and punctate cerebellar haemorrhages in $9 \%$ of the infants of this high-risk population. Previous studies have shown that small extra axial haemorrhages are a common MRI finding in asymptomatic term newborns. Looney et al. [17] reported a prevalence of $26 \%$ of asymptomatic intracranial haemorrhages on MRI after vaginal birth in full-term infants, the majority were subdural and infratentorial haemorrhages. Rooks et al. [18] reported an incidence of $20 \%$ of PF subdural haemorrhages in asymptomatic term infants. Most haemorrhages resolved spontaneously within one month and did not affect neurodevelopmental outcome at 2 years. Punctate cerebellar haemorrhages are a common MRI finding in very preterm infants and have a favourable outcome $[16,29]$. Not much is known about the incidence and consequences of punctate cerebellar haemorrhage in term infants. Our study suggests that these haemorrhages are also common in high-risk term infants.

Our study has a few limitations. Firstly, although prior studies demonstrated the clinical significance of posterior

Table. 3 Imaging findings in nine infants with discrepancies between CUS and MRI in the detection of major PF abnormality

\begin{tabular}{llll}
\hline Category & MF-CUS findings & MRI findings & No infants \\
\hline HIE & Normal & Abnormal SI in cerebellar vermis on DWI \\
CNS infection & Increased echogenicity cerebellum & Normal & 4 \\
CNS malformation & Normal & Cerebellar dysplasia & 1 \\
& Normal & Small arachnoid cyst in cerebello-pontine angle & 1 \\
PF haemorrhage & Normal & (Old) vermian haemorrhage on T2* MRI, & 1 \\
& AF-CUS cerebellar haemorrhage, & MRI nout atrophy & \\
& MF-CUS normal & & 1 \\
\hline
\end{tabular}


Fig. 7 Female infant born at 37 weeks gestational age, with severe perinatal asphyxia. CUS on third postnatal day with normal appearance of the cerebellar vermis (arrows) on the midsagittal $(A)$ and right coronal MF (B) views. (C) Diffusionweighted MRI (seventh postnatal day) shows diffusion restriction in the cerebellar vermis (arrow), with corresponding low signal on the ADC map [Figure. (D) arrow]
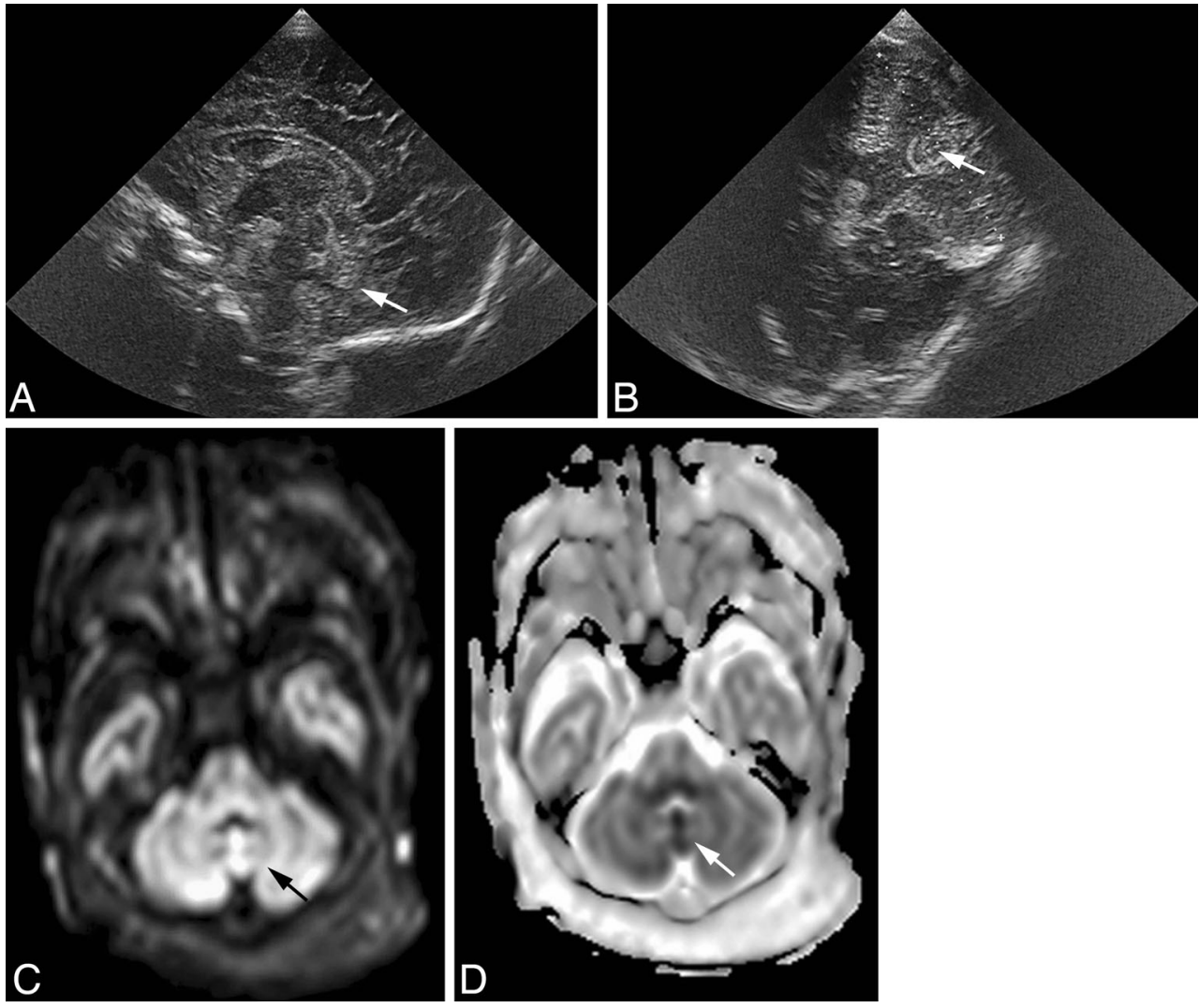

fossa abnormalities in preterm infants, clinical data in full term infants are limited. Therefore the importance of these findings, both on CUS and MRI, needs further investigation. Secondly, this was a selective and retrospective cohort of high-risk term infants assessed with both MF-CUS and MRI. The true incidence of PF abnormalities in specific clinical conditions, such as HIE, metabolic disorders or CNS infection, cannot be determined from our study and needs to be studied prospectively. In the third place, there was an interval between the CUS and MRI examinations. A prospective study with CUS and MRI performed on the same day may reveal a higher sensitivity of CUS, especially in cases with HIE $[30,31]$ or CNS infection. Finally, CUS examination is operator dependent. Increasing experience with the MF approach will improve knowledge of the normal anatomy and imaging appearance of the PF, and lead to an increased detection of abnormalities. However, all CUS images in this study were performed by well-trained neonatology staff members.

In conclusion, MF-CUS enables early diagnosis of PF abnormalities in high-risk, full-term neonates. Adding these MF images is relatively easy, can be performed bedside, and takes only a few minutes of extra imaging time. We therefore advocate to perform MF CUS not only in preterm, but also in highrisk term neonates, especially when MRI is not directly available.
Acknowledgments The scientific guarantor of this publication is Dr. G. Meijler, Department of Neonatology, Isala Hospital, Zwolle, The Netherlands. The authors of this manuscript declare no relationships with any companies, whose products or services may be related to the subject matter of the article. The authors state that this work has not received any funding. No complex statistical methods were necessary for this paper. Institutional Review Board approval was obtained. Written informed consent was waived by the Institutional Review Board. Ultrasound and MR images of three subjects included in the current cohort have been previously shown as examples in a practice guideline article by S.J. Steggerda et al. in Early Human Development, 88 (2012). All images in the current manuscript are the result of this original research and have not been published before. Methodology: retrospective, diagnostic or prognostic study, performed at one institution.

Open Access This article is distributed under the terms of the Creative Commons Attribution Noncommercial License which permits any noncommercial use, distribution, and reproduction in any medium, provided the original author(s) and the source are credited.

\section{References}

1. Limperopoulos C, Bassan H, Gauvreau K et al (2007) Does cerebellar injury in premature infants contribute to the high prevalence of long-term cognitive, learning, and behavioral disability in survivors? Pediatrics 120:584-593

2. Limperopoulos C, Robertson RL, Sullivan NR, Bassan H, du Plessis AJ (2009) Cerebellar injury in term infants: clinical characteristics, magnetic resonance imaging findings, and outcome. Pediatr Neurol 41:1-8 
3. Steggerda SJ, de Bruine FT, Smits-Wintjens VE, Walther FJ, van Wezel-Meijler G (2012) Ultrasound detection of posterior fossa abnormalities in full-term neonates. Early Hum Dev 88:233-239

4. Miall LS, Cornette LG, Tanner SF, Arthur RJ, Levene MI (2003) Posterior fossa abnormalities seen on magnetic resonance brain imaging in a cohort of newborn infants. J Perinatol 23:396-403

5. Volpe JJ (2008) Neurology of the newborn, 5th edn. Saunders, Philadelphia

6. Steinlin M, Blaser S, Boltshauser E (1998) Cerebellar involvement in metabolic disorders: a pattern-recognition approach. Neuroradiology 40:347-354

7. Leijser LM, de Vries LS, Rutherford MA et al (2007) Cranial ultrasound in metabolic disorders presenting in the neonatal period: characteristic features and comparison with MR imaging. AJNR Am J Neuroradiol 28:1223-1231

8. Williamson WD, Percy AK, Fishman MA et al (1985) Cerebellar hemorrhage in the term neonate: developmental and neurologic outcome. Pediatr Neurol 1:356-360

9. Steggerda SJ, Leijser LM, Walther FJ, van Wezel-Meijler G (2009) Neonatal cranial ultrasonography: how to optimize its performance. Early Hum Dev 85:93-99

10. Correa F, Enriquez G, Rossello J et al (2004) Posterior fontanelle sonography: an acoustic window into the neonatal brain. AJNR Am J Neuroradiol 25:1274-1282

11. Enriquez G, Correa F, Aso C et al (2006) Mastoid fontanelle approach for sonographic imaging of the neonatal brain. Pediatr Radiol 36:532-540

12. Di Salvo DN (2001) A new view of the neonatal brain: clinical utility of supplemental neurologic US imaging windows. Radiographics 21: 943-955

13. Steggerda SJ, Leijser LM, Wiggers-de Bruine FT, van der Grond GJ, Walther FJ, van Wezel-Meijler G (2009) Cerebellar injury in preterm infants: incidence and findings on US and MR images. Radiology 252:190-199

14. Meijler G (2012) Neonatal cranial ultrasonography, 2nd edn. Springer Berlin, Heidelberg

15. van Wezel-Meijler G, Leijser LM, de Bruine FT, Steggerda SJ, van der Grond GJ, Walther FJ (2009) Magnetic resonance imaging of the brain in newborn infants: practical aspects. Early Hum Dev 85:85-92

16. Steggerda SJ, de Bruine FT, van den Berg-Huysmans AA et al (2013) Small cerebellar hemorrhage in preterm infants: perinatal and postnatal factors and outcome. Cerebellum 12:794-801

17. Looney CB, Smith JK, Merck LH et al (2007) Intracranial hemorrhage in asymptomatic neonates: prevalence on MR images and relationship to obstetric and neonatal risk factors. Radiology 242:535-541
18. Rooks VJ, Eaton JP, Ruess L, Petermann GW, Keck-Wherley J, Pedersen RC (2008) Prevalence and evolution of intracranial hemorrhage in asymptomatic term infants. AJNR Am J Neuroradiol 29: 1082-1089

19. de Bruine FT, Steggerda SJ, van den Berg-Huysmans AA et al (2014) Prognostic value of gradient echo T2* sequences for brain MR imaging in preterm infants. Pediatr Radiol 44:305-312

20. Rutherford M, Ward P, Allsop J, Malamatentiou C, Counsell S (2005) Magnetic resonance imaging in neonatal encephalopathy. Early Hum Dev 81:13-25

21. Triulzi F, Parazzini C, Righini A (2006) Patterns of damage in the mature neonatal brain. Pediatr Radiol 36:608-620

22. Vermeulen RJ, Fetter WP, Hendrikx L, Van Schie PE, van der Knaap MS, Barkhof F (2003) Diffusion-weighted MRI in severe neonatal hypoxic ischaemia: the white cerebrum. Neuropediatrics 34:72-76

23. Martinez-Biarge M, Diez-Sebastian J, Kapellou O et al (2011) Predicting motor outcome and death in term hypoxic-ischemic encephalopathy. Neurology 76:2055-2061

24. Sargent MA, Poskitt KJ, Roland EH, Hill A, Hendson G (2004) Cerebellar vermian atrophy after neonatal hypoxic-ischemic encephalopathy. AJNR Am J Neuroradiol 25:1008-1015

25. Le Strange E, Saeed N, Cowan FM, Edwards AD, Rutherford MA (2004) MR imaging quantification of cerebellar growth following hypoxic-ischemic injury to the neonatal brain. AJNR Am J Neuroradiol 25:463-468

26. Connolly DJ, Widjaja E, Griffiths PD (2007) Involvement of the anterior lobe of the cerebellar vermis in perinatal profound hypoxia. AJNR Am J Neuroradiol 28:16-19

27. Jouvet P, Cowan FM, Cox P et al (1999) Reproducibility and accuracy of MR imaging of the brain after severe birth asphyxia. AJNR Am J Neuroradiol 20:1343-1348

28. Alderliesten T, Nikkels PG, Benders MJ, de Vries LS, Groenendaal F (2013) Antemortem cranial MRI compared with postmortem histopathologic examination of the brain in term infants with neonatal encephalopathy following perinatal asphyxia. Arch Dis Child Fetal Neonatal Ed 98:F304-F309

29. Tam EW, Rosenbluth G, Rogers EE et al (2011) Cerebellar hemorrhage on magnetic resonance imaging in preterm newborns associated with abnormal neurologic outcome. J Pediatr 158:245-250

30. Epelman M, Daneman A, Kellenberger CJ et al (2010) Neonatal encephalopathy: a prospective comparison of head US and MRI. Pediatr Radiol 40:1640-1650

31. Daneman A, Epelman M, Blaser S, Jarrin JR (2006) Imaging of the brain in full-term neonates: does sonography still play a role? Pediatr Radiol 36:636-646 